\title{
Extinction properties of the X-ray bright/optically faint afterglow of GRB 020405
}

\author{
G. Stratta ${ }^{1}$, R. Perna ${ }^{2,4}$, D. Lazzati ${ }^{2}$, F. Fiore ${ }^{3}$, L. A. Antonelli ${ }^{3}$, and M. L. Conciatore ${ }^{3}$ \\ ${ }^{1}$ LATT, Laboratoire d'Astrophysique Toulouse-Tarbes, 14 Av. Edouard Belin, 31400 Toulouse, France \\ e-mail: gstratta@ast.obs-mip.fr \\ 2 JILA and Department of Astrophysical and Planetary Sciences, University of Colorado at Boulder, 440 UCB, Boulder, \\ CO 80309, USA \\ 3 INAF - Osservatorio Astronomico di Roma, via Frascati 33, Monteporzio Catone, 00040, Rome, Italy \\ ${ }^{4}$ Department of Astrophysical Sciences, 4 Ivy Lane, Princeton, NJ 08544, USA
}

Received 25 April 2005 / Accepted 15 June 2005

\begin{abstract}
We present an optical-to-X-ray spectral analysis of the afterglow of GRB 020405. The optical spectral energy distribution not corrected for the extragalactic extinction is significantly below the X-ray extrapolation of the single powerlaw spectral model suggested by multiwavelength studies. We investigate whether considerable extinction could explain the observed spectral "mismatch" by testing several types of extinction curves. For the first time we test extinction curves computed with time-dependent numerical simulations of dust grains destruction by the burst radiation. We find that an extinction law weakly dependent on wavelength can reconcile the unabsorbed optical and X-ray data with the expected synchrotron spectrum. A gray extinction law can be provided by a dust grain size distribution biased toward large grains.
\end{abstract}

Key words. gamma-rays: bursts - ISM: dust, extinction

\section{Introduction}

Long-duration Gamma Ray Bursts (GRBs) are associated with the core collapse of massive stars exploding as type Ic supernovae (GRB 980425, Galama et al. 1998; GRB 021211, Della Valle et al. 2003; GRB 030329, Stanek et al. 2003; GRB 031203, Malesani et al. 2004). Due to their short lifetime, massive stars are likely to die within their birthplace. Consequently, long-duration GRBs occur in the same star formation region where their progenitor star was born and where it rapidly evolved. If high redshift galaxy star formation regions are akin to Galactic giant molecular clouds, a dense and dusty environment is expected in the vicinity of GRBs.

Indeed, the measured equivalent hydrogen column densities $N_{\mathrm{H}}$ obtained from X-ray afterglow spectral analysis are on average consistent with those observed along the line of sight of Galactic molecular clouds (e.g. Galama \& Wijers 2001; De Pasquale et al. 2003; Stratta et al. 2004). In some afterglows (e.g. GRB 971214, Ramaprakash et al. 1998; GRB 980703 Bloom et al. 1998; GRB 980329, Yost et al. 2002) a high visual extinction $A_{\mathrm{V}}$ in the GRB host galaxy has been measured. In addition, a large dust content was inferred from high resolution spectroscopy by refractory metal abundance analysis along the line of sight of four optical afterglows (Savaglio et al. $2003,2004)$. However, on average, the estimated afterglow reddening is low and the rest frame visual extinction is a factor 10-100 lower than expected from the extrapolation of the
X-ray $N_{\mathrm{H}}$, assuming the Galactic dust-to-gas ratio (Šimon et al. 2001; Galama \& Wijers 2000). In a few high redshift GRBs for which high resolution afterglow spectroscopy was performed, high $N_{\mathrm{H}}$ from $\mathrm{Ly}_{\alpha}$ absorption was measured. On the other hand for the same GRBs a low $A_{\mathrm{V}}$ was inferred from continuum absorption modeled with the Small Magellanic Cloud (SMC) extinction law. These results can be explained by a low metallicity and/or a low dust-to-gas ratio in the burst environment (e.g. Hjorth et al. 2003; Vreeswijk et al. 2004).

Alternatively, the GRB environment may be characterized by a "non-standard" extinction law (neither Galactic nor SMC; Stratta et al. 2004; Savaglio et al. 2004). In particular, an extinction law weakly dependent on wavelength can provide high visual extinction without substantial reddening (e.g. Hjorth et al. 2003). Dust properties of high redshift environments - such as the GRB host galaxies - are still poorly known. We have some indications from the Magellanic Clouds (e.g. Pei et al. 1992) and from a sample of local starburst galaxies (e.g. Calzetti et al. 1997) and Active Galactic Nuclei (e.g. Maiolino et al. 2001; Maiolino 2004) that the dust composition, grain size distribution and dust to gas ratio can be significantly different from the ones observed in our Galaxy. The GRB star-forming environment, in addition, may not be representative of the typical low-density host galaxy inter-stellar matter (ISM). Finally, the intense X-ray and UV flux from the burst itself can modify the intrinsic dust grain size distribution by differential dust grain destruction, since sublimation processes are more effective on 
small grains (e.g. Waxman \& Draine 2000; Fruchter et al. 2001; Draine \& Hao 2002; Perna \& Lazzati 2002; Perna et al. 2003).

A diagnostic tool of the GRB environment extinction properties is the simultaneous optical-to-X-ray afterglow continuum spectral analysis. The intrinsic optical continuum can be extrapolated from the X-ray data and dust absorption can be measured from the deviations of the measurements from the expected fluxes. Good quality data are necessary to constrain the free parameters of the continuum model (the position of the cooling break possibly laying between the optical and X-ray bands). This method was applied, with a various degree of accuracy, in a handful of GRBs (Stratta et al. 2004).

In this work we present a study of the optically faint/X-ray bright afterglow of GRB 020405 (e.g. Dado et al. 2002; Masetti et al. 2003; Bersier et al. 2003; Covino et al. 2003; Price et al. 2003; Berger et al. 2003; Mirabal et al. 2003). This burst was discovered by the IPN on 2002 April 5.029 UT. The duration of the burst was $\sim 40 \mathrm{~s}$, the $25-100 \mathrm{keV}$ fluence was $\sim 3 \times$ $10^{-5} \mathrm{erg} \mathrm{cm}^{-2}$ and the peak flux was $\sim 10^{-6} \mathrm{erg} \mathrm{cm}^{-2} \mathrm{~s}^{-1}$. This GRB was also observed by the GRB Monitor on board BeppoSAX with a duration of $\sim 60 \mathrm{~s}$ in the $40-700 \mathrm{keV}$ band and a 50-700 keV fluence of $4 \times 10^{-5} \mathrm{erg} \mathrm{cm}^{-2} \mathrm{~s}^{-1}$ (e.g. Price et al. 2003). Optical observations started $18 \mathrm{~h}$ after the burst event (Price et al. 2003) for a period of $\sim 10$ days. An unknown fading source was localized at RA $13^{\mathrm{h}} 58^{\mathrm{m}} 03^{\mathrm{s}} .12$ and Dec $-31^{\circ} 22^{\prime} 22^{\prime \prime} .2$ with an uncertainty of 0.3 (Masetti et al. 2002; Price et al. 2003). An exhaustive summary of all the afterglow observations performed in the optical band is presented in Masetti et al. (2003). The host galaxy has redshift $z=0.691 \pm 0.002$ (Masetti et al. 2003). Chandra observations started on April 6.711 UT and lasted until 7.350 UT using LETGS in conjunction with the ACIS detector and revealed a new fading source that was identified as the X-ray afterglow counterpart. Mirabal et al. (2003) found a featureless spectrum, well described by a power law continuum with energy spectral index $^{1} \alpha_{\mathrm{X}}=0.72 \pm 0.21$ and a rest frame $N_{\mathrm{H}}$ of $(4.7 \pm 3.7) \times$ $10^{21} \mathrm{~cm}^{-2}$. The X-ray light curve decayed as a power law, with a temporal decay index ${ }^{1} \delta_{X}=1.97 \pm 1.10$ (Mirabal et al. 2003). The optical (VRI) decay index is $\delta_{\mathrm{O}}=1.54 \pm 0.06$ from 1 to 10 days after the burst (Masetti et al. 2003). The NIR bands ( $J$ and $H$ ) show a less steep decay with index $\delta_{\mathrm{IR}} \sim 1.3$ (Masetti et al. 2003). Radio observations show a rapidly fading "radio flare" at 1.2 days from the burst event (Berger et al. 2003). Berger et al. (2003) found that the radio, optical and X-ray data are self-consistently modeled assuming a collimated ejecta expanding into a uniform medium. Best fit parameters yield a jet break time at about 1 day after the burst and indicate that the cooling frequency at that time is at energies lower than the optical band. The same conclusion on the location of the cooling frequency was obtained by Masetti et al. (2003), who showed how the optical spectral energy distribution has a break around the $J$ band. For these reasons, for this burst there is convincing evidence for the absence of a spectral break between the X-ray and the optical band. The different decay index and spectral slope observed in the NIR band with respect to the optical may be due to the presence of the cooling frequency between the

\footnotetext{
1 Where the afterglow flux is described as $F \propto v^{-\alpha} t^{-\delta}$.
}

optical and the NIR bands (Masetti et al. 2003; Berger et al. 2003).

The paper is organized as follows: in Sect. 2 we summarize the data analysis procedure; in Sect. 3 we describe the adopted extinction curves; in Sects. 4 and 5 we present and discuss our results.

\section{Data analysis procedure}

We followed the standard procedure for the X-rays (0.1-10.0 keV) data reduction for Chandra ACIS data. We grouped energy channels in order to have at least 20 counts per bin in order to apply the $\chi^{2}$ statistic in the fitting procedure. We first fit the X-rays spectrum with a power law model and two absorber components. The column density of the first absorber was fixed to the Galactic value toward the line of sight of the burst $4.3 \times 10^{20} \mathrm{~cm}^{-2}$ (Dickey \& Lockmann 1995). We subsequently allowed for the second absorber a free column density to constrain a possible contribution of absorption from the host galaxy ISM. We found that the data are well fitted by this model, with a best fit energy spectral index of $\alpha_{\mathrm{X}}=1.0 \pm 0.2$ and rest frame $(z=0.691)$ hydrogen column density of $N_{\mathrm{Hz}}=(0.8 \pm 0.2) \times 10^{22} \mathrm{~cm}^{-2}$ in addition to the Galactic absorption $\left(\chi^{2} /\right.$ d.o.f. $=22.3 / 24$, where d.o.f. stands for degrees of freedom). The best fit model (absorbed power law) $1.6-10.0 \mathrm{keV}$ flux is $8.7 \times 10^{-13} \mathrm{erg} \mathrm{cm}^{-2} \mathrm{~s}^{-1}$. Errors are at $1 \sigma$ level. We note that our spectral index is steeper than, but still consistent with, the one obtained by Mirabal et al. (2003).

The optical-NIR SED was obtained by taking the photometric points from the journal table published by Masetti et al. (2003). Observations in each photometric band $(U, B, V, R, I$, $J, H$ and $K$ ) were performed at different times and with different telescopes. We extrapolated the magnitudes at the observational epoch $t_{0}$ of $7.01 \mathrm{UT}$ (1.98 days after the burst). To this purpose, we firstly selected the observations performed as closer as possible to $t_{0}$, and then we extrapolated the magnitude at $t_{0}$ assuming a single powerlaw with decay index estimated at that time from Masetti et al. (2003). The decay index uncertainty was propagated to the magnitude errors with an average increase that depends on the temporal distance of the photometric measure from $t_{0}$ (see Table 1 in Masetti et al. 2003). This results in an increase of the error on the magnitudes of $20 \%$ or more, depending on the temporal distance from the selected $t_{0}$. We choose $t_{0}$ as the average time of the X-ray observations given the large uncertainty in the X-ray decay slope (Mirabal et al. 2003).

We corrected the magnitudes for Galactic extinction $(E(B-V)=0.054 \mathrm{mag})$ using the Galactic dust infrared map by Schlegel et al. (1998). Assuming a total-to-selective extinction $R_{\mathrm{V}}=A_{\mathrm{V}} / E(B-V)=3.1$, we derived the extinction value in each photometric band from the Galactic extinction curve parameterization $A(\lambda) / A_{\mathrm{V}}$ by Cardelli et al. (1989). We finally converted magnitudes to fluxes using the effective wavelengths and normalization fluxes given by Fukugita et al. (1995). A 7\% systematic error was added quadratically to the magnitude errors to account for mag-to-flux conversion uncertainties and for intercalibration errors from different telescopes (see also 
Table 1. The optical-near infrared magnitudes of the optical afterglow of GRB 020405 (from Masetti et al. 2003), corrected for Galactic extinction and rescaled at $t_{0}=\mathrm{UT} 7.01\left(T_{\mathrm{GRB}}+1.98\right.$ days $)$.

\begin{tabular}{cccccccc}
\hline \hline$U$ & $B$ & $V$ & $R$ & $I$ & $J$ & $H$ & $K$ \\
\hline $21.22 \pm 0.12$ & $21.90 \pm 0.1$ & $21.50 \pm 0.08$ & $21.08 \pm 0.06$ & $20.56 \pm 0.12$ & $19.37 \pm 0.10$ & $18.72 \pm 0.10$ & $17.99 \pm 0.10$ \\
\hline
\end{tabular}

Masetti et al. 2001). The resulting magnitudes are summarized in Table 1.

A simultaneous fit of the NIR-to-X-rays spectrum was performed with the X-ray spectrum corrected for the total photoelectric absorption and the optical SED corrected for the Galactic extinction but not corrected for an extra-Galactic component. For the intrinsic SED, we considered both a single powerlaw and a broken powerlaw, the latter with break energy below the optical range. In order to estimate the host galaxy extinction we multiplied the continuum spectral model by an absorption component. We tested different ISM dust composition and dust to gas ratios by assuming several type of extinction curves (see Sect. 3).

\section{Host galaxy extinction}

Since we do not know a priori the host galaxy extinction curve, we tested several types of environments (Fig. 1) with different dust grain size distributions, dust compositions and dust to gas mass ratios. The ratio between the hydrogen column density $N_{\mathrm{H}}$ and the visual extinction $A_{\mathrm{V}}$ provides a measure of the dust-to-gas ratio for a given dust distribution and composition. For simulated time-dependent extinction laws, the dust-to-gas ratio at the relevant time is derived from the simulations.

We tested the following extinction curves:

- the Galactic extinction curve (hereafter G) from Cardelli et al. (1989), for which $N_{\mathrm{H}} / A_{\mathrm{V}}=0.18 \times 10^{22} \mathrm{~cm}^{-2}$ (Predehl \& Schmidt 1995).

- The Small Magellanic Cloud extinction curve (hereafter SMC) from Pei et al. (1992), for which $N_{\mathrm{H}} / A_{\mathrm{V}}=1.6 \times$ $10^{22} \mathrm{~cm}^{-2}$ (Weingartner \& Draine 2000).

- The attenuation curve derived for a sample of local starburst galaxies (hereafter C) from Calzetti et al. (1994). No $N_{\mathrm{H}} / A_{\mathrm{V}}$ has been measured for this case due to the complexity of the geometry of the dust and star distribution inside these galaxies (Calzetti et al. 2001).

- Two extinction curves obtained by Maiolino et al. (2001) for the environment of a sample of AGNs. The Q1 extinction curve is computed assuming a power law dust grain size distribution $\mathrm{d} n(a) \propto a^{-q} \mathrm{~d} a$ in the range $a_{\min }=$ $0.005 \mu<a<a_{\max }=10 \mu$ and $q=-3.5$. In this case $N_{\mathrm{H}} / A_{\mathrm{V}}=0.7 \times 10^{22} \mathrm{~cm}^{-2}$. The Q2 extinction curve is derived assuming $a_{\min }=0.005 \mu<a<a_{\max }=1 \mu$ and $q=-2.5$ and it yields $N_{\mathrm{H}} / A_{\mathrm{V}}=0.3 \times 10^{22} \mathrm{~cm}^{-2}$.

- Extinction curves resulting from numerical simulations performed with the code of Perna \& Lazzati (2002). This code computes the temporal evolution of the dust grain size distribution, taking into account grain erosion due to UV and X-ray illumination. The ionizing continuum is tailored to the case of GRB 020405 (see above). Only the 40 s of the

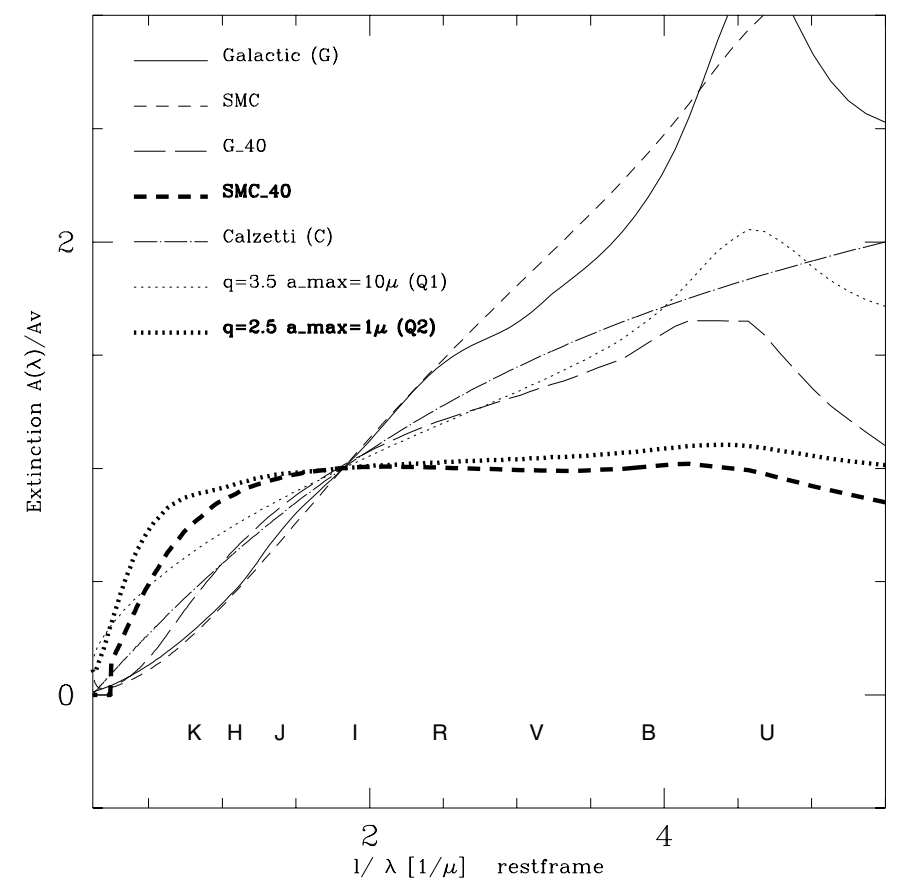

Fig. 1. Extinction curves adopted in the analysis of the optical-NIR photometry against $1 / \lambda$ where $\lambda$ is the rest frame wavelength. We note that the weakest wavelength dependence is shown by the Q2 extinction curve and by the SMC-modified extinction curve computed by dust destruction numerical simulations (SMC_40, Perna et al. 2003). We also indicate the position of the photometric filters for a system located at $z=0.695$, the redshift of GRB 020405. Extinction curves that provide an acceptable fit to the data are emphasized with thicker lines.

burst emission were considered, since the overall fluence of the later afterglow was smaller than that of the prompt emission by at least an order of magnitude. Initial conditions were either a Galactic (final curve labelled G_40) or SMC (final curve labelled SMC_40) ISM. A uniform cloud with radius $R=7 \times 10^{22} \mathrm{~cm}$ and density $n=4 \times$ $5 \mathrm{~cm}^{-3}$ was considered. We checked that changing this setup would not change our conclusions.

\section{Results}

We fit the NIR/optical and the X-ray spectral energy distribution, extrapolated to a common epoch (see Sect. 2), with a dust-absorbed power law model. The NIR-to-X-ray spectral index $\alpha$ was let free to vary. We found that the optical/NIR data corrected for the "standard" extinction curves, namely the G and the SMC, are incompatible with X-ray data (see Table 2). Unacceptable fits were obtained also with the Calzetti, the Q1 and the G_40 curves (see Sect. 3). 

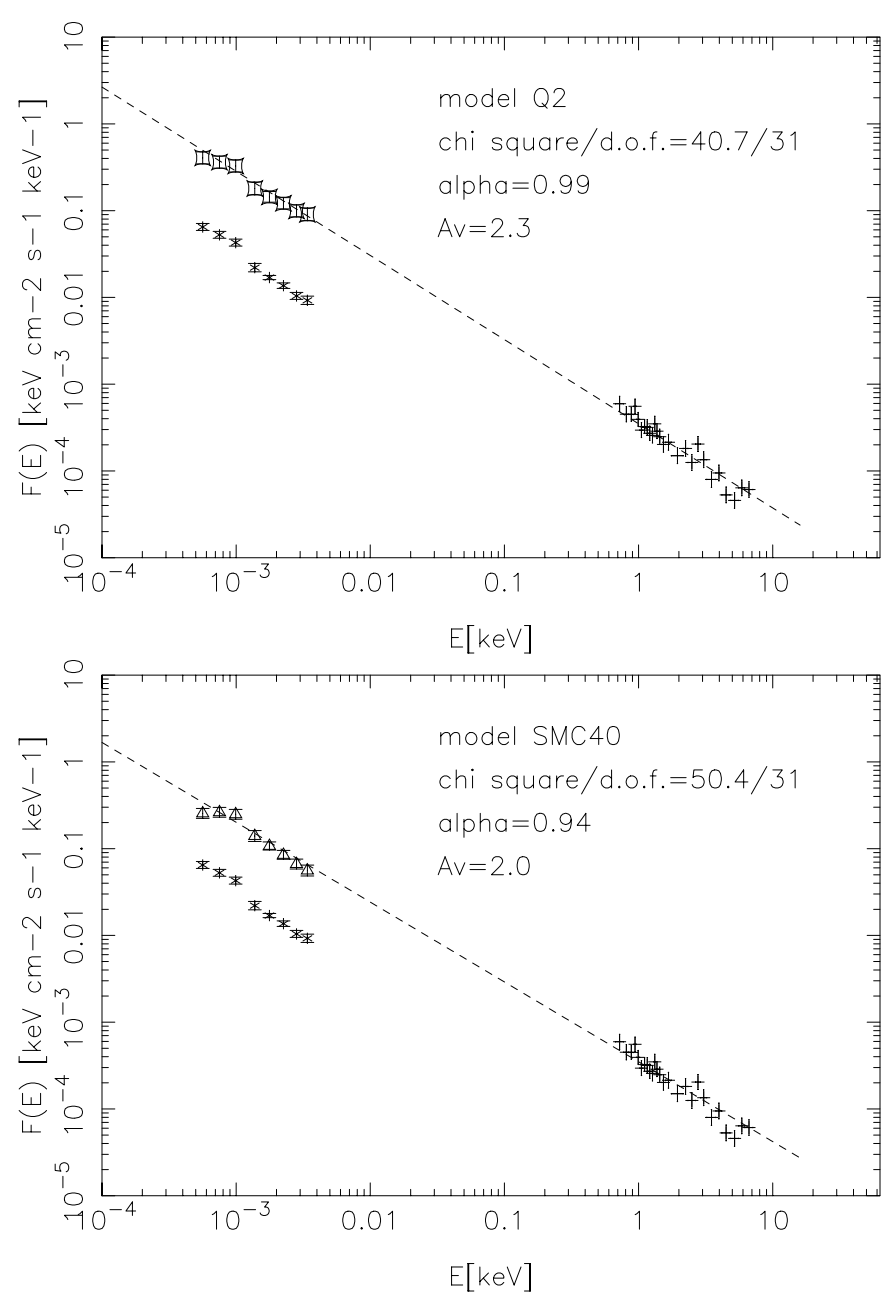

Fig. 2. NIR-to-X-ray absorbed power-law fits to the data of GRB 020405. A Q2 absorption curve is used in the upper panel, while a SMC_40 curve is used in the bottom panel. Optical data are shown both uncorrected (asterisks) and corrected (squares and triangles) for the host galaxy extinction. See Table 2 for the best fit parameters.

Table 2. Best fit parameters from simultaneous NIR-to-X-ray spectral analysis assuming a power law model and different extinction curves to evaluate rest frame host galaxy extinction $A_{\mathrm{V}}$ (see Sect. 3).

\begin{tabular}{lccc}
\hline \hline $\begin{array}{l}\text { Extinction } \\
\text { curve }\end{array}$ & $\alpha$ & $\begin{array}{c}A_{\mathrm{V}} \\
\mathrm{mag}\end{array}$ & $\chi^{2} /$ d.o.f. \\
\hline $\mathrm{G}$ & $0.75 \pm 0.01$ & $0.36 \pm 0.04$ & $89.3 / 31$ \\
\hline SMC & $0.74 \pm 0.01$ & $0.33 \pm 0.04$ & $83.8 / 31$ \\
SMC_40 & $0.94 \pm 0.02$ & $2.0 \pm 0.1$ & $50.4 / 31$ \\
\hline C & $0.80 \pm 0.02$ & $0.78 \pm 0.08$ & $66.5 / 31$ \\
Q1 & $0.79 \pm 0.01$ & $0.69 \pm 0.08$ & $76.7 / 31$ \\
Q2 & $0.99 \pm 0.02$ & $2.27 \pm 0.14$ & $40.7 / 31$ \\
\hline
\end{tabular}

Acceptable fits with comparable probability $P\left(>\chi^{2}\right)$ of $15 \%$, were instead obtained with the Q2 and the SMC_40 curves. These curves have the weakest dependence on wavelengths among those considered (see Fig. 1). We note that the relatively high $\chi^{2}$ levels are due to the apparent bend of the continuum in the NIR which cannot be reproduced by a power-law (Fig. 2).
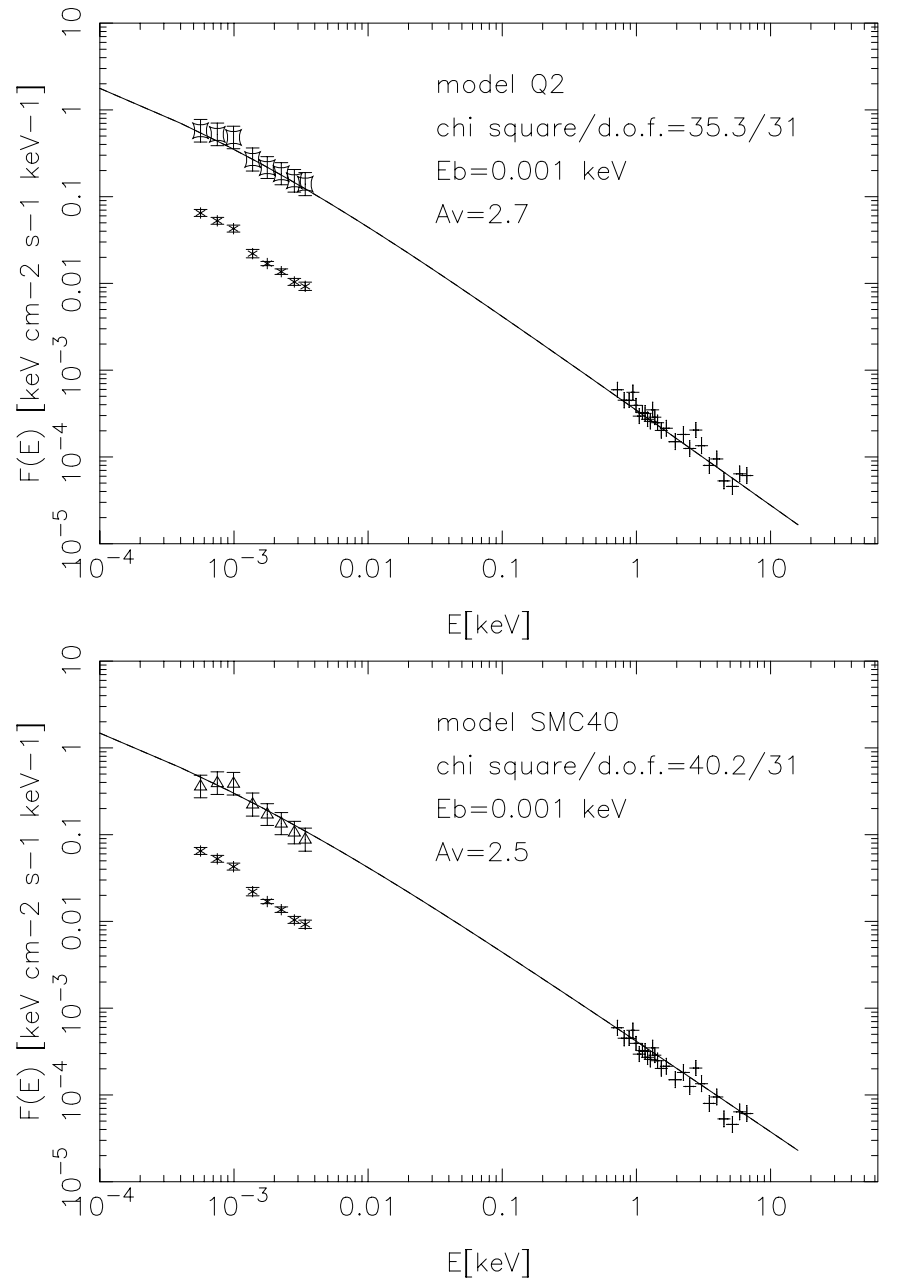

Fig. 3. Same as Fig. 2 but with a smoothed broken power-law continuum model (see Table 3).

We tested therefore a smoothed broken power law spectrum (with the sharpness parameter $s$ equal to 1, Granot \& Sari 2002) with break energy between the optical and NIR bands. The optical-to-X-rays spectral index was let free to vary within the $90 \%$ confidence level interval $0.8-1.0$, found from the $\mathrm{X}$-ray analysis. Assuming $v_{\mathrm{c}}<v_{\mathrm{X}}$, from the X-ray spectral slope we derive an electron spectral index $p=2.0 \pm 0.4$. We then compute the expected NIR spectral slope (corresponding to $(p-1) / 2$ for $v_{\mathrm{NIR}}<v_{\mathrm{c}}$, Sari et al. 1998) that we let free to vary within the $90 \%$ confidence level interval (0.3-0.7). Even allowing for this extra degree of freedom, we found that the $G$, SMC, C, Q1 and G_40 absorption models are inconsistent with the data (see Table 3).

For the models Q2 and SMC_40 we find an improvement in the fit with a $2.5 \sigma$ statistical significance, according to the F-test (Fig. 3). They both fit the data successfully (Table 3). We then checked the consistency of the measured $A_{\mathrm{V}}$ values with the $N_{\mathrm{H}}$ measured from the X-ray analysis and the $N_{\mathrm{H}} / A_{\mathrm{V}}$ relationship expected (see Sect. 3). For the Q2 model, we found $N_{\mathrm{H}}=0.7 \times 10^{22} \mathrm{~cm}^{-2}$ assuming a power law model and $N_{\mathrm{H}}=$ $0.8 \times 10^{22} \mathrm{~cm}^{-2}$ assuming a broken power law. These $N_{\mathrm{H}}$ values are consistent with the $N_{\mathrm{H}}\left[(0.8 \pm 0.2) \times 10^{22} \mathrm{~cm}^{-2}\right]$ measured from X-ray analysis. For the SMC_40 model, even though the 
Table 3. Best fit parameters from simultaneous X-ray and optical spectral analysis assuming a smoothed broken power law model $(s=$ 1 , from Granot \& Sari 2002) with the break energy between the optical and the NIR bands (see Sect. 2). We assumed different extinction curves to evaluate rest frame host galaxy extinction $A_{\mathrm{V}}$.

\begin{tabular}{lccc}
\hline \hline $\begin{array}{l}\text { Extinction } \\
\text { curve }\end{array}$ & $\begin{array}{c}E_{\text {break }} \\
\mu\end{array}$ & $\begin{array}{c}A_{\mathrm{V}} \\
\mathrm{mag}\end{array}$ & $\chi^{2} /$ d.o.f. \\
\hline $\mathrm{G}$ & not found & - & - \\
\hline SMC & not found & - & - \\
SMC_40 & $1.12 \pm 0.20$ & $2.53 \pm 0.3$ & $40.2 / 31$ \\
\hline $\mathrm{C}$ & $1.3 \pm 0.5$ & $0.87 \pm 0.11$ & $64.5 / 31$ \\
Q1 & not found & - & - \\
Q2 & $1.26 \pm 0.20$ & $2.7 \pm 0.3$ & $35.3 / 31$ \\
\hline
\end{tabular}

shape of the extinction curve fits the data well, a gray extinction with $A_{\mathrm{V}} \gtrsim 2$ is obtained only for $N_{\mathrm{H}} \sim 10^{24} \mathrm{~cm}^{-2}$, largely exceeding the X-ray measured $N_{\mathrm{H}}$. Formally a self consistent fit can be obtained assuming a dust-to-gas ratio 100 times larger than the SMC one. This would imply a $\gtrsim 10$ times solar metallicity. We consider such physical conditions too extreme, even tough GRB (star formation) environments are expected to be especially dusty.

\section{Discussion}

We have studied the broad-band spectrum of the afterglow of GRB 020405 from NIR to X-ray bands. The optical-NIR and $\mathrm{X}$-ray spectral indices are mutually consistent. However, this burst is peculiar since it has an optical-NIR flux a factor of $\gtrsim 10$ times dimmer than the extrapolation of the X-ray spectrum. This can be attributed to an inverse Compton component in the X-ray band (Sari \& Esin 2001). Alternatively, the optical brightness and spectrum could be affected by dust extinction. We investigated the latter possibility. We found a selfconsistent model that allowed us to constrain the properties of the dust distribution and its formation history.

From a simultaneous NIR-to-X-ray spectral analysis, we found that the optical data can be fit with the external shock model only if corrected for an extinction curve weakly dependent on wavelength. Such an extinction curve can be the result of small dust grains coagulation and/or dust grain destruction (e.g. Maiolino et al. 2001; Perna et al. 2003). In Fig. 1 we plot the extinction curves derived from these two different processes (see Sect. 3). The remarkable similarity of these extinction curves, computed assuming different processes, is evident.

The dust coagulation rate increases with density $\propto n^{1 / 2}$ (Draine 1985). It is therefore favored in dense environments such as the cores of star formation regions where long GRBs are expected to explode. Incidentally, extinction curves weakly dependent on wavelengths have been inferred also for other extra-galactic objects, such as AGNs (Maiolino et al. 2001).

A dust distribution biased towards large grains can also be due to grain destruction mechanisms (Waxman \& Draine 2000; Fruchter et al. 2001; Draine \& Hao 2002; Perna \& Lazzati 2002). We have attempted to reproduce the observations starting from known dust distributions in the local Universe (Galactic and SMC). The evolution of the initial distributions is followed as a result of the grain interaction with the burst ionizing flux (Perna et al. 2003). We find that the modified Galactic distribution cannot reproduce the observations, while the SMC one can reproduces them if an extremely large dust-to-gas ratio and metallicity are assumed. Since these conditions are hardly realized in nature, a scenario in which the large grain distribution is intrinsic to the host galaxy is favored.

The difficulty in reproducing the results as a consequence of dust destruction can be understood since dust destruction reduces the opacity besides modifying the reddening law. Since this burst requires a large extinction on a day timescale, it is likely that the opacity is provided by dust at a large distance from the burster, which is not affected by the burst photons. Prompt optical-NIR observations are better suited for the detection of dust destruction, since the evolution is caught "on the act". This would allow us to detect dust before it is destroyed and, based on the properties of what is left, infer the geometrical and physical conditions of the host galaxy ISM (Perna et al. 2003).

Acknowledgements. The authors thank the referee F. J. Castander for his useful comments on the manuscript. G.S. is supported by the Research Training Network Gamma-Ray Bursts: an Enigma and a Tool funded by the EU.

\section{References}

Berger, E., Soderberg, A., Frail, D. A., \& Kulkarni, F. R. 2003, ApJ, 587, L5

Bersier, D., McLeod, B., Garnavich, P. M., et al. 2003, ApJ, 583, L63

Bloom, J. S., Frail, D. A., Kulkarni, S. R., et al. 1998, ApJ, 508, 21

Calzetti, D. 1997, AJ, 113, 1

Calzetti, D., Kinney, A. L., \& Storchi-Bergmann, T. 1994, ApJ, 429, 582

Cardelli, J. A., Clayton, G. C., \& Mathis, J. S. 1989, ApJ, 345, 245

Covino, S., Malesani, D., Ghisellini, G., et al. 2003, A\&A, 400, L9

Dado, S., Dar, A., \& De Rujula, A. 2002, A\&A, 393, L25

Della Valle, M., Piro, L., Perna, R., et al. 2003, A\&A, 406, 33

De Pasquale, M., Vreeswijk, P. M., van Paradijs, J., et al. 2003, ApJ, 592,1018

Dickey, J. M., \& Lockman, F. J. 1990, ARA\&A, 28, 215

Draine, B. T. 1985, Protostars and Planets II, ed. D. C. Black, \& M. S. Matthews, Sp. Sc. Ser. (The Univ. of Arizona Pr.)

Draine, B. T., \& Hao, L. 2002, ApJ, 569, 780

Fruchter, A. S., Krolik, J. H., \& Rhoads, J. S. 2001, ApJ, 563, 597

Fukugita, M., Shimasaku, K., \& Ichikawa, T. 1995, PASP, 107, 945

Galama, T. J., \& Wijers, A. M. J. 2001, ApJ, 549, L209

Galama, T. J., Vreeswijk, P. M., van Paradijs, J., et al. 1998, Nature, 395,670

Granot, J., \& Sari, R. 2002, ApJ, 568, 820

Hjorth, J., Møller, P., Gorosabel, J., et al. 2003, ApJ, 597, 699

Maiolino, R., Marconi, A., \& Oliva, E. 2001, A\&A, 365, 37

Maiolino, R., Schneider, R., Oliva, E., et al. 2004, Nature, 431, 533

Malesani, D., Tagliaferri, G., Chincarini, G., et al. 2004, ApJ, 609, L5

Masetti, N., Palazzi, E., Pian, E., et al. 2001, A\&A, 374, 382

Masetti, N., Palazzi, E., Pian, E., et al. 2003, A\&A, 404, 465

Mirabal, N., Paerels, F., \& Halpern, J. P. 2003, ApJ, 587, 128

Pei, Y. C. 1992, ApJ, 395, 130

Perna, R., \& Lazzati, D. 2002, ApJ, 580, 261 
Perna, R., Lazzati, D., \& Fiore, F. 2003, ApJ, 585, 775

Predehl, P., \& Schmitt, J. H. M. M. 1995, A\&A, 293, 889

Price, P. A., Kulkarni, S. R., Berger, E., et al. 2003, ApJ, 589, 838

Ramaprakash, A. N., Kulkarni, S. R., Frail, D. A., et al. 1998, Nature, 393,43

Sari, R., \& Esin, A. A. 2001, ApJ, 548, 787

Sari, R., Piran, T., \& Narayan, R. 1998, ApJ, 197, L17

Savaglio, S., \& Fall, M. F. 2004, ApJ, 614, 293

Savaglio, S., Fall, M., \& Fiore, F. 2003, ApJ, 585, 638

Schlegel, D. J., Finkbeiner, D. P., \& Davis, M. 1998, ApJ, 500, 525
Šimon, V., Hudec, R., Pizzichini, G., \& Masetti, N. 2001, A\&A, 377, 450

Stanek, K. Z., Matheson, T., Garnavich, P. M., et al. 2003, ApJ, 591, 17

Stratta, G., Fiore, F., Antonelli, L. A., Piro, L., \& De Pasquale, M. 2004, ApJ, 608, 846

Vreeswijk, P., Ellison, S. L., Ledoux, C., et al. 2004, A\&A, 419, 927

Waxman, E., \& Draine, B. T. 2000, ApJ, 537, 796

Weingartner, J. C., \& Draine, B. T. 2000, A\&AS, 197, 4207

Yost, S. A., Frail, D. A., Harrison, F. A., et al. 2002, ApJ, 577, 155 